\title{
Bleomycin-induzierte Flagellantendermatitis
}

\section{Bleomycin induced Flagellate Dermatitis}

\section{Zusammenfassung}

Wir berichten über einen 43-jährigen Patienten, der sich bei pulmonaler Metastasierung eines malignen Keimzelltumors einer Polychemotherapie mit Bleomycin, Etoposid und Cisplatin unterziehen musste. Unmittelbar nach Einleitung der Behandlung entwickelte sich eine ausgeprägte Flagellantendermatitis. Nachdem daraufhin Bleomycin durch Ifosfamid ersetzt wurde, konnte bei den nachfolgenden Behandlungszyklen kein Rezidiv der Dermatose beobachtet werden. Die Flagellantendermatitis, benannt nach den Büßern des Mittelalters, den Flagellanten, die sich durch Geißeln strichförmige Verletzungen zufügten, ist als Nebenwirkung einer Chemotherapie bisher nur bei Bleomycin beschrieben worden. Pathogenetisch lässt auch die hier vorgestellte Kasuistik einen direkten toxischen Effekt des Zytostatikums vermuten.

\section{Abstract}

We report on a 43 years old patient who had to undergo a polychemotherapy consisting of bleomycin, etoposide and cisplatine due to a malignant germ cell tumor with accompanying pulmonal metastasis. Immediately after therapy initiation an extensive flagellate dermatitis developed. Then, bleomycin was replaced by ifosfamide, and no further dermatitis was observed during the subsequent treatment cycles. Fagellate dermatitis, named after the medieval penitents - the Flagellants , who selfinflicted streaky injuries by whipping themselves, is a side effect of a chemotherapy which has been up to now described only for bleomycin. This case report corroborates the assumption that pathogenetically this cystostatic drug has a direct toxic effect.

\section{Einleitung}

Bleomycin, ein Vertreter der zytostatisch wirksamen Antibiotika, besteht aus einem Gemisch basischer Glykoproteide, das erstmals 1965 von Umezawa aus Streptomyces verticillus isoliert werden konnte [1]. Als typische Behandlungsindikationen gelten Plattenepithelkarzinome der Haut und Schleimhäute, Lymphome und Hodentumore. Die pharmakologische Wirkung des Bleomycins beruht dabei auf einer Störung des Einbaus von Thymidin in die DNA, wodurch deren Synthese blockiert und die Zellteilung verhindert wird [2,3]. Die enzymatische Inaktivierung erfolgt durch eine Hydrolase, deren jeweilige Gewebekonzentrationen in der Haut und der Lunge deutlich erniedrigt sind [4]. Dies erklärt einerseits die selektive Anreicherung in den beiden
Organsystemen, andererseits aber auch die hier gehäuft auftretenden Nebenwirkungen. Während pulmonal die interstitielle Fibrose als die typische Nebenwirkung einer Bleomycin-Therapie angesehen wird, die bei bis zu 12\% der behandelten Patienten auftritt und pathogenetisch als Folge einer Verbrauchskoagulopathie interpretiert wird, findet sich an der Haut ein klinischmorphologisch und pathogenetisch unterschiedliches Spektrum von Nebenwirkungen, von denen einige als außerordentlich typisch, andere sogar als substanzspezifisch bezeichnet werden [5-7]. Hierzu zählen neben Hyperpigmentierungen und der Bleomycin-induzierten Pseudosklerodermie auch die Flagellantendermatitis, die ein unverwechselbares klinisches Bild bietet, wie dies auch die nachfolgende Kasuistik belegt. 


\section{Anamnese}

Im Zusammenhang mit einem operativ entfernten malignen Keimzelltumor des linken Hodens waren bei den Staging-Untersuchungen des hier vorgestellten 43-jährigen Patienten multiple Metastasen der Lungen nachgewiesen worden. Zur Behandlung der pulmonalen Metastasierung wurde eine Chemotherapie nach dem PEB-Schema eingeleitet, wobei der Patient $30 \mathrm{mg}$ Bleomycin an den Tagen 1, 8 und 15 sowie $220 \mathrm{mg}$ Etoposid und $44 \mathrm{mg}$ Cisplatin an den Tagen $1-5$ erhalten sollte. Bereits am zweiten Tag des ersten Zyklus klagte er jedoch über einen quälenden Pruritus, der ihn zu heftigem Kratzen veranlasste. Weitere zwei Tage später wurden erstmals persistierende, teilweise auch nässende Rötungen im Bereich der Kratzspuren beobachtet, woraufhin die Chemotherapie zunächst abgebrochen werden musste.

\section{Dermatologischer Befund}

Am Stamm lateral und dorsal im Bereich der LWS-Region, gluteal, an den Oberschenkeln und an den Unterarmen kratzspurenartige, parallel verlaufende, über der LWS-Region auch flächenhaft konfluierende, unscharf begrenzte, infiltrierte Erytheme mit umschrieben auftretenden, stecknadelkopfgroßen Papulovesikeln und Krusten. Daneben gluteal, im Bereich des Gesichtes und am behaarten Kopf disseminiert stehende, stecknadelkopf- bis halbkirschkerngroße, derbe, erythematöse Papeln und Knoten (Abb. 1-3).

\section{Histopathologischer Befund}

Zur histopathologischen Untersuchung wurden Biopsien aus den strichförmigen Erythemen gluteal und den erythematösen Papeln des Gesichtes entnommen. Dabei zeigten sich histopathologisch identische Befunde. Bei unauffälliger Epidermis fanden sich im Bereich der gesamten Dermis perivaskulär angeordnete Infiltrate, bestehend aus Lymphozyten und Eosinophilen.

\section{Laborbefunde}

Vorübergehende, deutlich ausgeprägte Leuko- und Thrombozytopenie bei sonst unauffälligen routinemäßig erhobenen Laborparametern.

\section{Therapie und Verlauf}

Die Behandlung erfolgte mit Antihistaminika und initial $40 \mathrm{mg}$ Prednisolon täglich, entsprechend einer Dosis von $0,5 \mathrm{mg} / \mathrm{kg}$ Körpergewicht. Auf eine höhere Prednisolondosis war im Hinblick auf die Leukozytopenie und die damit verbundene Infektionsgefahr verzichtet worden. Allerdings wurden zusätzlich topische Glukokortikoide verordnet. Bei schrittweiser Reduktion der oralen Prednisolondosis bildete sich der akut entzündliche Befund innerhalb von zwei Wochen auffällig langsam zurück, wobei sich nun eine zunehmende Hyperpigmentierung ausbildete (Abb.4). Die Chemotherapie wurde sieben Wochen nach dem abgebrochenen ersten Zyklus wieder aufgenommen. Anstelle des Bleomycins erhielt der Patient $2640 \mathrm{mg}$ Ifosphamid unter Beibehaltung der genannten Dosierung von Etoposid und Cisplatin. Ein Rezidiv der Flagellantendermatitis konnte anschließend nicht mehr beobachtet werden.

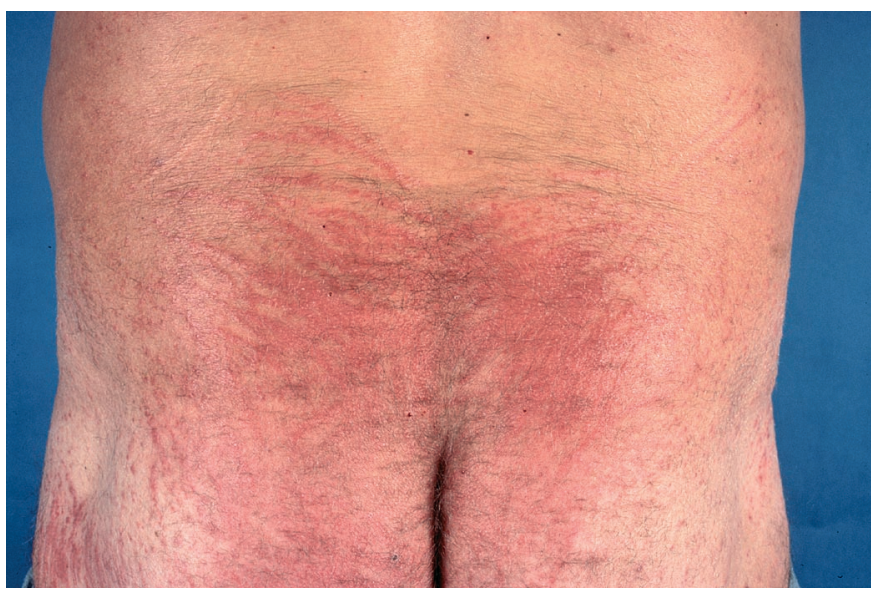

Abb. 1 Strichförmige, konfluierende Erytheme, LWS-Region.

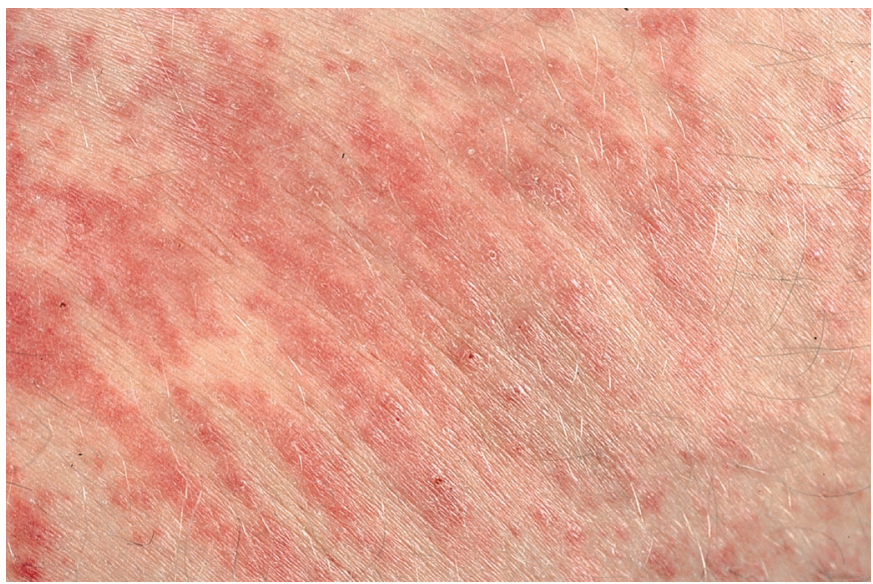

Abb. 2 Strichförmige Erytheme im Bereich von Kratzspuren, Oberschenkel rechts.

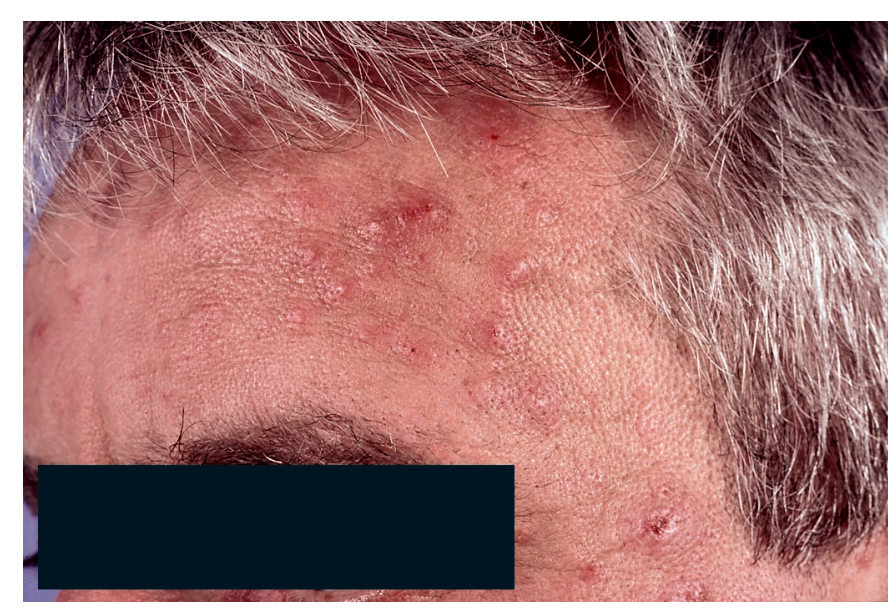

Abb. 3 Erythematöse Papeln und Knoten des Gesichtes.

\section{Diskussion}

Die Durchführung einer Bleomycin-Therapie kann mit zahlreichen, unterschiedlich schwer wiegenden Nebenwirkungen verbunden sein, von denen die Lungenfibrose besonders gefürchtet ist, da sich einerseits keine sichere Beziehung zwischen der im 


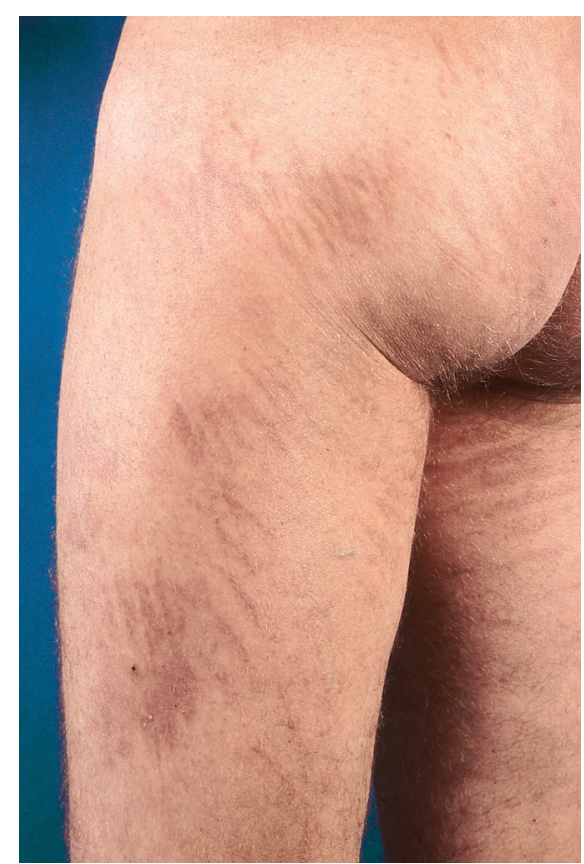

Abb. 4 Strichförmige Hyperpigmentierungen vier Wochen nach Bleomycin-Gabe.

Einzelfall verordneten Bleomycin-Dosis und dem Auftreten der pulmonalen Komplikationen herstellen lässt und andererseits der einmal eingeleitete Prozess der Fibrosierung auch nach Abbruch der Bleomycin-Therapie weiter fortschreiten kann. Obwohl die Wahrscheinlichkeit einer klinisch relevanten Lungentoxizität im Allgemeinen erst bei einer kumulativen Dosis von mehr als $400 \mathrm{mg}$ Bleomycin gegeben ist, wurden auch letal verlaufende lungentoxische Reaktionen bei Bleomycin-Dosierungen unter $100 \mathrm{mg}$ beobachtet $[5,8-10]$. Somit lässt sich keine Schwellendosis definieren, deren Einhaltung das Auftreten von Nebenwirkungen sicher ausschließen würde. Dies gilt im Übrigen auch für kutane Nebenwirkungen einer Bleomycin-Therapie. Dabei wird die Mehrzahl der dermatologischen Nebenwirkungen bei kumulativen Dosierungen zwischen 100 und $300 \mathrm{mg}$ Bleomycin beobachtet [11]. Für die Häufung der Nebenwirkungen in diesem Dosisbereich mag dabei von Bedeutung sein, dass die genannten Dosierungen bei den verschiedenen Indikationen als therapeutisch angesehen und somit in den meisten Behandlungsfällen appliziert werden. In Einzelfällen finden sich jedoch auch entsprechende kutane Nebenwirkungen bei sehr viel niedrigeren, häufig dann auch nur einmalig gegebenen BleomycinDosierungen, z. B. zwischen 15 und $30 \mathrm{mg}[12,13]$.

Darüber hinaus sind auch die prozentualen Angaben zur Häufigkeit der verschiedenen kutanen Nebenwirkungen sehr unterschiedlich und werden mit $8-24 \%, 55 \%$ und $83 \%$ angegeben [14-16]. Dabei darf vermutet werden, dass diese doch deutlich voneinander abweichenden Prozentangaben auf unterschiedliche Applikationsformen, Einzeldosen und Gesamtdosierungen der jeweils ausgewerteten Patientengruppen zurückzuführen sind. Auch die fachspezifische Betrachtungsweise und die damit verbundene gezielte Suche nach dermatologischen Nebenwirkungen dürfte als ein weiterer Grund für die unterschiedlichen Prozentangaben anzusehen sein [11].

Die dermatologischen Nebenwirkungen einer Bleomycin-Therapie umfassen ein weites Spektrum klinisch-morphologischer
Krankheitsbilder. Als häufigste Nebenwirkung wird dabei ein diffuser Haarverlust angesehen, der ausschließlich am Capillitium auftritt und sich nach Beendigung der Therapie innerhalb von Wochen und Monaten zurückbilden kann [11]. Auch die Entwicklung erythematöser, teilweise deutlich infiltrierter, lichenifizierter oder hyperkeratotischer Papeln und Plaques im Bereich der mechanisch belasteten Regionen, z.B. an den Ellenbogenund Kniegelenken, gilt als häufige und besonders typische $\mathrm{Ne}$ benwirkung einer Bleomycin-Therapie. Dabei kann es zur Ausbildung von schmerzlosen Ulzerationen kommen, die ohne Narbenbildung abheilen. Auch die im Verlauf zunehmende Hyperpigmentierung dieser Papeln und Plaques wird als typisch charakterisiert $[11,17]$. Eine weitere Besonderheit sind die auffällig zahlreichen dermatologischen Nebenwirkungen im Bereich der Hände und Füße. Hierzu zählen palmoplantar auftretende schwielenartige Hyperkeratosen, paraunguale Erytheme, meist schmerzlose Schwellungen der kleinen Finger- und Zehengelenke, Akrozyanose, Fingerspitzennekrosen und eine RaynaudSymptomatik [11,17-19]. Nagelveränderungen, meist in Form streifenförmiger oder flächenhafter Hyperpigmentierungen, werden hingegen weniger häufig beschrieben [20,21]. Die akral lokalisierte oder auch generalisiert auftretende Pseudosklerodermie zählt ebenfalls zu den typischen kutanen Komplikationen einer Bleomycin-Therapie. Im Gegensatz zu den bisher aufgeführten Nebenwirkungen wurde die Bleomycin-induzierte Pseudosklerodermie jedoch nur vereinzelt beobachtet [22-24]. Das klinische Bild ist gekennzeichnet durch flächenhaft indurierte Fibrosierungen, morpheaartige Atrophien und wiederum ausgeprägte Hyperpigmentierungen. Innere Organsysteme sind von dem Krankheitsprozess üblicherweise nicht betroffen; entsprechend fehlen auch die für die progressive systemische Sklerodermie spezifischen Laborparameter. Die dermatologischen Veränderungen können sich im Übrigen nach Beendigung der Bleomycin-Therapie vollständig zurückbilden.

Die ungewöhnlichste Form einer durch Bleomycin verursachten Nebenwirkung ist jedoch zweifellos die Flagellantendermatitis, die auch als flagellatartige Hyperpigmentierung oder als Flagellatendermatitis bezeichnet wird $[25,26]$. Da sich die Namensgebung jedoch auf die mittelalterlichen Büßer als Personen, die Flagellanten, bezieht, ist der Begriff Flagellantendermatitis zutreffender [27]. Das klinische Bild der Flagellantendermatitis ist unverwechselbar und als Nebenwirkung einer Arzneimitteltherapie bisher substanzspezifisch nur bei Bleomycin beschrieben worden. Als auslösende Dosierung werden in der Literatur einmalige oder kumulative Dosen zwischen 15 und 400 mg Bleomycin angegeben $[13,25,28]$. Nach einem initial auftretenden Pruritus kommt es in den meisten Fällen unmittelbar nach Einleitung einer Bleomycin-Therapie oder während der ersten Behandlungszyklen zu strichförmig infiltrierten Erythemen, teilweise mit dicht stehenden Papeln. Die strichförmigen, 20-30 cm langen Morphen sind parallel angeordnet und treten in den Kratzspuren auf, die sich die Patienten aufgrund des ungewöhnlich quälenden Pruritus selbst zufügen. Bei exzessivem Kratzen konfluieren die Erytheme zu flächenhaften Arealen, wobei der ursprünglich striäre Charakter in den Randbereichen der Herde erhalten bleibt. Bevorzugt betroffen sind in symmetrischer Verteilung die Unterarme, die Oberschenkel, die Glutealregionen und der seitliche Stamm. Nach Abbruch der Bleomycin-Therapie bilden sich die Erytheme innerhalb einiger Wochen langsam wie- 
der zurück, wobei es gleichzeitig zu einer Umwandlung in strichförmige Hyperpigmentierungen kommt, die Monate persistieren können [12,13,25, 26, 28].

Die histopathologischen Befunde der Flagellantendermatitis sind weitgehend unspezifisch. Bei unauffälliger Epidermis oder hier auch herdförmig auftretender Spongiose finden sich in der Dermis perivaskulär angeordnete, lymphozytäre Infiltrate mit einzelnen Eosinophilen. Die teilweise wandverdickten Kapillargefäße können einen partiellen oder totalen Verschluss ihrer Lumina aufweisen. Darüber hinaus zeigt sich eine intraepidermale Pigmentierung im Stratum basale mit oder ohne ausgeprägte Pigmentinkontinenz $[12,25,28]$.

Die Pathogenese der Flagellantendermatitis ist nicht geklärt. Neben allergischen Mechanismen ist auch eine durch den zerfallenden Tumor ausgelöste Reaktion für möglich gehalten worden [28]. Das vereinzelte Auftreten der Dermatose unmittelbar nach Einleitung einer Bleomycin-Therapie, das auch bei dem hier vorgestellten Patienten beobachtet werden konnte, die gehäuften Exazerbationen bei einer kumulativen Dosis von 100-200 mg Bleomycin und auch die histopathologischen Gefäßveränderungen sprechen jedoch hier für einen toxischen Mechanismus $[25,28,29]$. Der bei den einzelnen Patienten möglicherweise unterschiedlich ausgeprägte protrahierte enzymatische Abbau des Bleomycins könnte dabei die Beobachtung erklären, dass die Flagellantendermatitis durch sehr unterschiedliche Bleomycin-Dosierungen ausgelöst werden kann. Bei einem sehr langsamen Abbau des Bleomycins würde somit bereits eine kleine Dosierung ausreichen, um eine Flagellantendermatitis auszulösen. Ein weiterer pathogenetischer Mechanismus ist offensichtlich die mechanische Irritation der Haut. Berücksichtigt man die histopathologisch nachgewiesenen Gefäßschäden, könnten diese durch das üblicherweise ausgeprägte Kratzen zunehmen und die damit verbundenen entzündlichen Reaktionsabläufe weiter verstärkt werden. Allerdings ist die mechanische Provokation nur für die striäre Anordnung der entzündlichen Infiltrate verantwortlich. Zusätzlich können sich auch außerhalb der Kratzspuren Morphen entwickeln, wie dies auch bei unserem Patienten beobachtet wurde, der besonders im Bereich des Gesichtes entzündliche Papeln und Knoten entwickelt hatte, die klinisch als papulöses Arzneimittelexanthem imponierten. Das Auftreten entzündlicher Morphen außerhalb mechanisch malträtierter Hautareale ist auch von anderen Autoren beschrieben worden [28]. Die für viele Bleomycin-induzierte Nebenwirkungen am Hautorgan typischen Hyperpigmentierungen sind in ihrer Pathogenese ebenfalls nicht geklärt. Diskutiert werden eine Stimulation der MSHSekretion, ein direkter Effekt des Bleomycins auf die Melaninsynthese und postinflammatorische Mechanismen [25, 28,30].

Zur Behandlung der Flagellantendermatitis werden Antihistaminika sowie je nach Schweregrad topische oder systemische Glukokortikoide empfohlen. Allerdings, auch hierfür ist unser Patient ein typisches Beispiel, ist nur mit einer langsamen Rückbildung der dermatologischen Befunde zu rechnen.
${ }^{1}$ Umezawa H. Bleomycin and other antitumor antibiotics of high molecular weight. Antimicrob Agents Chemother 1965; 5: 1079-1085

${ }^{2}$ Gilmartin ME, Epner S, Lasky L, Freedberg IM. Bleomycin effects upon mammalian epidermal DNA synthesis. Acta Derm Venereol (Stockh) 1977; $57: 373-378$

${ }^{3}$ Umezawa H. Structure and action of bleomycin. Prog Biochem Pharmacol 1976; 11: $18-27$

${ }^{4}$ Bennet JM, Reich SD. Bleomycin. Ann Int Med 1979; 90: 945-948

${ }^{5}$ Burkhardt A, Gebbers JO, Höltje WJ. Die Bleomycin-Lunge. Dtsch med Wschr 1977; 102: $281-289$

${ }^{6}$ Fritsch H, Drings P, Fournier DV. Klinische Erfahrungen mit Bleomycin bei verschiedenen metastasierten Tumoren. In: v. Wilmanns W (Hrsg). Bleomycin, experimentelle Grundlagen und erste klinische Ergebnisse. Laupheim: Ahnen KG, 1975

${ }^{7}$ Höltje WJ, Burkhardt A, Gebbers JO, Maerker R. Intraarterielle Bleomycintherapie von Plattenepithelkarzinomen der Mundhöhle. Z Krebsforsch 1976; 88: 69-90

${ }^{8}$ Comis RL. Bleomycin pulmonary toxicity. In: Carter CK (Hrsg). Bleomycin, current status and new developments. New York: Acad. Press, 1978

${ }^{9}$ Frey F, Marone C, Zimmermann A. Interstitielle Pneumopathie nach niedrig dosierter Bleomycintherapie. Schweiz med Wschr 1977; 107: $1418-1422$

${ }^{10}$ Huntington MC, Du Priest RW, Fletcher WS. Intraarterial bleomycin therapy in inoperable squamous cell carcinomas. Cancer (Philad) 1973; 31: $153-157$

${ }^{11}$ Kiefer O. Über die Nebenwirkungen der Bleomycintherapie auf der Haut. Dermatologica 1973; 146: 229-243

12 Fernandez-Obregon AC, Hogan KP, Bibro MK. Flagellate pigmentation from intrapleural bleomycin. J Am Acad Dermatol 1985; 13: 464-468

${ }^{13}$ Polla BS, Merot Y, Saurat JH, Slosman D. Flagellate pigmentation from bleomycin. J Am Acad Dermatol 1986; 14: 690

${ }^{14}$ Franz G, Kaether M, Krämer U. Erfahrungen bei der Behandlung gynäkologischer Malignome mit Bleomycin. In: v. Wilmanns W (Hrsg). Bleomycin, experimentelle Grundlagen und erste klinische Ergebnisse. Laupheim: Ahnen KG, 1975

${ }^{15}$ Kleibel F, Becker J. Zweijährige klinische Erfahrungen mit Bleomycin. In: v. Wilmanns W (Hrsg). Bleomycin, experimentelle Grundlagen und erste klinische Ergebnisse. Laupheim: Ahnen KG, 1975

${ }^{16}$ Rathert P, Terhorst B, Lutzeyer W. Bleomycin bei malignen Genitaltumoren des Mannes. Urologie 1974; 13: 67-71

${ }^{17}$ Werner Y, Törnberg B. Cutaneous side effects of bleomycin therapy. Acta Derm Venereol (Stockh) 1976; 56: 155-158

${ }^{18}$ Malcolm D. Bleomycin-induced injury to the hands. J med Soc New York 1978; 75: $314-316$

${ }^{19}$ Scheulen ME, Schmidt CG. Raynaud-Syndrom. In: Eckhardt S, Holzner HJ, Nagel GA (Hrsg). Beiträge zur Onkologie: Wirkung und Nebenwirkungen von Bleomycin. Basel: Karger, 1982

${ }^{20}$ Perrot H, Ortonne JP. Hyperpigmentation after bleomycin therapy. Ultrastructural study. Arch Dermatol Res 1978; 261: 245-252

${ }^{21}$ Shelty MR. Case of pigmented banding of the nail caused by bleomycin. Cancer Treat Rep 1977; 61: 501 - 502

22 Behrens S, Reuther T, von Kobyletzki G, Kastner U, Dirschka T, von Kerscher M, von Altmeyer P. Bleomycin-induzierte PSS-artige Pseudosklerodermie. Hautarzt 1998; 49: 725-729

${ }^{23}$ Bork K, Korting GW. Symptomatische Sklerodermie durch Bleomyzin. Hautarzt 1983; 34: 10-12

${ }^{24}$ Kerr LD, Spiera H. Scleroderma in association with the use of bleomycin: a report of 3 cases. J Rheumatol 1992; 19: 294-296

${ }^{25}$ Albig J, Gollnick H, Detmar M, Orfanos CE. Flagellatartige Hyperpigmentierung durch Bleomycin. Hautarzt 1992; 43: 376-379

${ }^{26}$ Haas N, Wittmann G, Bock A, Czarnetzki BM. Flagellatendermatitis nach einmaliger, niedrig dosierter Bleomycingabe. Z Dermatol 1994; 180: $26-28$

${ }^{27}$ Haas N, Vogt R, Sterry W. Shiitake-Dermatitis. Hautarzt 2001; 52: $132-135$

${ }^{28}$ Altmeyer P, Schäfer G, Reimer R, Kachel HG. Ein Beitrag zur Pathogenese der kutanen Bleomycin-Nebenwirkungen. Akt Dermatol 1984; 10: $191-196$

${ }^{29}$ Lazar AP, Lazar P. Streaky pigmentation in a patient with AIDS. Cutis 1988; 42: 397-398

${ }^{30}$ Bernd A, Altmeyer P, Marsch H, Schäfer G, Holtzmann A. Bleomycin enhances the tyrosinase activity of human malignant melanoma cells in culture. Pharmacol Res Commun 1986; 18: 1075-1091 